\title{
Anemia and upper digestive tract at the Yalgado Ouédraogo university hospital in Ouagadougou, Burkina Faso
}

\begin{abstract}
Introduction: The World Health Organization has classified anemia as one of the ten most serious health problems of the modern world. The detection of anemia should lead to a precise etiologic assessment supported by clinical and biological data.

Objective: To describe the epidemiological and diagnostical characteristics of upper digestive tract pathologiesin non-sickle cell disease anemic patients at the Ouédraogo Yalgado university hospital in Ouagadougou.

Patients and methods: This was a prospective and cross- sectional study carried out from March 1,2016 to July 31, 2017 in the Department of clinical hematology, diagnostic and interventional endoscopy and pathological anatomy and cytology of the aforementioned university hospital. The study involved all non-sickle cell patients above 15 years' old who were seen in hematology consultation for anemia.

Results: The study involved a total of 58 patients including 42 women with a sex ratio of 0.4. Age ranged from 17 to 87 years with a mean age of 36.9. Most of the patients (44 cases out of 58) had iron-deficiency microcytic anemia. There were 5 cases of macrocytic anemia: vitamin B12 deficiency with 3 cases of Biermer's disease and 2 cases of vitamin B9 deficiency. We observed a prevalence of grade I peptic esophagitis $(50.99 \%)$ in esophageal pathology, a predominance of gastropathies (81.25\%) in gastric pathology and a predominance of bulbar ulcers $(60 \%)$ in duodenal pathology. Histology revealed chronic gastritis in all patients and chronic atrophic gastritis with the presence of pylori $\mathrm{H}$ in 53 out of 58 cases $(91.38 \%)$.
\end{abstract}

Conclusion: Upper digestive tract diseases diagnosed through endoscopy and histology (in particular pylori $\mathrm{H}$ ) can cause iron-deficiency anemia.

Keywords: anemia, upper digestive tract, Pylori H, Burkina Faso
Volume 8 Issue 2 - 2020

\author{
Koulidiati J, ${ }^{1-3}$ Sawadogo S, ${ }^{4}$ Kabore D, ' \\ Nikiema/Minoungou $M,{ }^{3}$ Some O R, ${ }^{4}$ Some \\ D M, ${ }^{5}$ Kafando $E,{ }^{2}$ BougmaA ${ }^{2}$ \\ 'Department of Clinical Hematology, Yalgado Ouédraogo \\ University Hospital, Burkina Faso \\ ${ }^{2}$ Unit of Training and Research in Health Sciences, University \\ Joseph Ki-Zerbo, Burkina Faso \\ ${ }^{3}$ Department of Biological Hematology Laboratory, Yalgado \\ Ouédraogo University Hospital, Burkina Faso \\ ${ }^{4}$ Institute of Health Sciences, Nazi Boni University, Burkina Faso \\ ${ }^{5}$ Department of Hepato-gastroenterology, Yalgado Ouédraogo \\ University Hospital, Burkina Faso
}

\section{Correspondence: Koulidiati Jérôme, Department of Clinical Hematology, Yalgado Ouédraogo University Hospital, Ouagadougou, Burkina Faso, 03 BP 7022 Ouagadougou 03 Burkina Faso,Email koulidiatij@yahoo.com \\ Kafando Eleonore, Assistant professor, Clinical Hematology, Department of Health Sciences, University Joseph Ki-Zerbo, 03 BP 702I Ouagadougou 03 Burkina Faso, Tel (+226) 253070 64/65,Fax (+226) 253072 42), Email ekafando@hotmail.com}

Received: April II, 2020 | Published: April 27, 2020

\section{Introduction}

Anemia is defined as a decrease in hemoglobin level below the normal lower levels of hemoglobin regardless of any variation in the plasma volume (hemodilutionorhemoconcentration). According to Beutler E and colleagues, normal lower levels of hemoglobin vary with age, sex, ethnicity, altitude and stage of pregnancy. ${ }^{1}$ The World Health Organization defined anemia by similar standards with moderate variations for those below 15 years old as shown in Table $1 .^{2}$

Table I Definition of anemia according to age and sex by the $\mathrm{WHO}^{2}$

\begin{tabular}{l}
\hline Age and sex \\
\hline Child 6 months to 5 years \\
Child 5 to II years \\
Child 12 to 14 years \\
Non pregnant women (>I5 years) \\
Pregnant women \\
Men (> 15 years)
\end{tabular}

g, gram;dL, deciliter
Anemia is a major public health issue that affects physical growth,cognitive development, reproduction and physical work capacity resulting in impaired human performance. ${ }^{1}$ The World Health Organization has classified anemia as one of the ten most serious health issues of the modern world which is also the most prevalent type of micronutrient deficiency in the world. Indeed, anemia affects about 2 billion people worldwide with a prevalence of $24.8 \%, 9$ out of 10 of whom live in countries with limited resources. The most vulnerable people are infants, children undergoing intensive growth, the elderly and pregnant women., ${ }^{1,3}$

The detection of anemia should lead to a precise etiologic assessment supported by clinical and paraclinical data. Anemia or rather anemias are the results of various and complex physiopathological causes and mechanisms which render some diagnoses intricate and challenging. Indeed, at one-year-old, only $30 \%$ of anemic patients present an etiological diagnosis. Iron, folates and vitamin B12 deficiencies are the main causes for anemia. ${ }^{1}$ Indeed, these micronutrients (iron, folates or vitamin B9, vitamin B12 or cobalamins) represent the three necessary exogenous factors for erythropoiesis.

Iron deficiency anemia or martial deficiency anemia is associated to chronic blood loss,often of gynecological origin in women during 
periods ofgenital activity and digestive activity in men. This explains how digestive endoscopy is important in that it is the main test for etiological diagnosis. ${ }^{4}$ In children, anemia is often related to intake deficiency. ${ }^{4}$

Vitamin B12 deficiency is a common condition, especially in the elderly. ${ }^{5}$ It can lead to serious hematological and neurological complications in the absence of early diagnosis. ${ }^{5}$ In adults, the syndrome of non-dissociation of vitamin B12 from its carrier protein and Biermer's disease are the two main causes of vitamin B12 deficiency. In Biermer's disease, gastric endoscopy with biopsy and histological examination revealsa chronic atrophic fundic gastritis of autoimmune origin. ${ }^{5}$

To the best of our knowledge, there has not been any study on the association between anemic patients and pathologies of the upper digestive tract in our country. Our objective is to describe the epidemiological and diagnostic characteristics in anemic patients at the Yalgado Ouedraogo University Hospital (CHU/YO) in Ouagadougou, Burkina Faso.

\section{Patients and methods}

We conducted a prospective and cross-sectional study on the patients who consulted for anemia in the Department of Clinical Hematology, Diagnostic and Interventional Endoscopy, and Pathological Anatomy and Cytology of the YalgadoOuedraogoUniversity Hospital in Ouagadougou (Burkina Faso). The study covered the period from March 1, 2016 to July 31, 2017.

The study included all patients with anemia due to iron deficiency or resistance to oral iron therapy and vitamin B12 deficiency from unidentified causes, who consented to the study. An initial blood count was performed in all patients and a second blood count was performed after Pylori $H$ eradication treatment in patients with Pylori $H$.Ferritinemia, serum iron was measured in microcytic anemia: mean corpuscular volume $(\mathrm{MCV})<80$ femtolitres (fL).

Anemia was classified as iron deficient when serum iron and ferritinemia were low.The test for vitamins B9, B12, anti-intrinsic factor antibody was carried out in the event of macrocytic anaemia: $\mathrm{MCV}>100 \mathrm{fL}$.

Each patient underwent an upper GI endoscopy. The patients had eaten their last meal latest 8 p.m. the day before, resulting in a gastric emptying of at least 8 hours. At the endoscopy, the patient's upper GI tract was explored in axial vision and then in retro-vision when the endoscope was removed. Five (5) biopsies were performed, two (2) in the body, one (1) at the angulus and two (2) in the antrum. The samples were sent in three (3) different tubes (corresponding to the three biopsy sites) to the laboratory of pathological anatomy and cytology of the Yalgado OUEDRAOGO University Hospital.

H. pylori in feces test was carried out after a treatment to eradicate the bacteria. Two protocols were used for treatment: sequential treatment and triple therapy based on the treatment recommendations for Pylori $H$ in adults according to the French Helicobacter Study Group 6 .

The following patients were excluded:

i. patients with a known cause of anemia (fibroma, menorrhagia...),

ii. pregnant and nursing women, iii. those infected with the human immunodeficiency virus (HIV),

iv. sickle cell patients,

v. those who've taken antibiotics in the last four weeks,

vi. and those who were receiving proton pump inhibitors (PPIs) in the last two weeks preceding the upper GI endoscopy.

We collected data using a survey form which included patient's marital status, socio-demographic characteristics, history, reason for consultation, biological characteristics of anemia, results of upper digestive endoscopy, histological examinations of biopsies, hemogram, pylori $H$ in stool test after eradication treatment for this bacterium.

Data was collected and analyzed on a microcomputer using Epi info 7. Statistical comparisons were made using chi2 test with a significance level $p<0.05$.

\section{Operational definitions:}

The diagnostic criteria for anemia were the ones used by the WHO, which is hemoglobin:

$<13 \mathrm{~g} / \mathrm{dLin}$ men,

and $<12 \mathrm{~g} / \mathrm{dLin}$ non-pregnant women.

Serum iron was considered low when it was $<12 \mu \mathrm{mol} / \mathrm{L}$. Ferritinemia was interpreted as low in females during genital activity for a level $<20 \mu \mathrm{g} / \mathrm{L}$, and in postmenopausal women and men for a level $<30 \mu \mathrm{g} / \mathrm{L}$.Vitamin B12 deficiency has been defined by a serum vitamin $\mathrm{B} 12$ level $<140$ picomoles/litre $(\mathrm{pmol} / \mathrm{L})$. The normal value for serum folate was $10.4-42.4 \mathrm{nmol} / \mathrm{L}$ or $4-18 \mathrm{mg} / \mathrm{L}$; the normal value for erythrocyte folate was $340 \mathrm{nmol} / \mathrm{L}$ or $150 \mathrm{mg} / \mathrm{L}$. The Intrinsic Factor Ac was found to be positive at $>1.54 \mathrm{AU} / \mathrm{mL}$ (reagents used: Intrinsic Factor Ab, Beckman Coulter).

\section{Results}

During the period of the study, we collated a total of 58 patients in the three aforementioned departments of the YalgadoOuedraogo University Hospital, corresponding to a recruitment rate of 40.94 cases/year.

The age of patients ranged from 17 to 87 years with a mean of 36.9 years; the majority of our patients, 44 cases/58(75.9\%) aged between 20 and 49 years. The population under study consisted of 42 females $(72.4 \%)$ and 16 males $(27.6 \%)$ with a sex ratio of 0.4 . There were more women in all age groups except in those aged 60 and over. Of the 58 patients recruited, $55(94.83 \%)$ had clinical manifestations of anemia. Asthenia was found in 27 patients/58 (46.55\%). Asthenia, pallor, dizziness, dyspnea and neurosensory signs (tinnitus, paresthesias) were observed in our patients.

Iron deficiency anemia was predominant in all age groups, especially in women under 50 years of age (Table 2); the distribution of the patients according to age, of the sex, of the hemoglobin rate, of the ferritin and the serum B12 is specified to the Table 2.

All five patients with macrocytic anemia had serum vitamin B12 levels lowered to less than $200 \mathrm{pg} / \mathrm{mL}$ with the presence of intrinsic factor antibodies in 3 of them; folic acid levels were lowered to less than $5 \mu \mathrm{g} / \mathrm{L}$ in two of them.Of the 58 anemic patients, nine patients had a normal upper GI endoscopy. 
Table 2 Distribution of the patients according to age, the sex, the complete blood count and the biochemistry

\begin{tabular}{|c|c|c|c|c|}
\hline Age and sex & $\begin{array}{l}\text { Number of } \\
\text { cases }\end{array}$ & Middle rate of hemoglobin $(\mathrm{g} / \mathrm{dL})$ & Middle ferritin $(\mu \mathrm{g} / \mathrm{ml})$ & Serum B I 2 (pmol/L) \\
\hline \multirow{2}{*}{ Women $<50$ years } & 27 & 9.47 & 7.29 & \\
\hline & 4 & 12.95 & 37.9 & \\
\hline \multirow{2}{*}{ Men $<50$ years } & 5 & 9.77 & 9.01 & \\
\hline & I & 10.14 & & 73 \\
\hline \multirow{3}{*}{50 to 70 years:Women } & 5 & 8.78 & 8.33 & \\
\hline & I & 13.06 & 38.71 & \\
\hline & 2 & 9.23 & & 70 \\
\hline \multirow{3}{*}{50 to 70 years : Men } & 4 & 9.19 & 15.48 & \\
\hline & I & 13.78 & & \\
\hline & 1 & 10.45 & & 71 \\
\hline \multirow{2}{*}{ Women $>70$ years } & I & 8.99 & 18.03 & \\
\hline & I & 12.99 & 45.89 & \\
\hline \multirow{3}{*}{ Men $>70$ years } & I & 13.79 & 41.6 & \\
\hline & 2 & 8.34 & 12.79 & \\
\hline & 1 & 9.97 & & 69 \\
\hline
\end{tabular}

$p=0.15$

Peptic esophagitis (26 cases) and mycotic esophagitis (01 case) represented 27 cases $/ 51$ cases, i.e. $52.94 \%$ of the esophageal pathologies found in our patients. The majority of grade I peptic esophagitis ( 21 women/26 or $80.77 \%$ ) were female. The majority of hiatal hernias (18 women/22 or $81.81 \%$ ) were female. Only women ( 2 cases) had endobrachyesophagus. Half ( 22 cases/44 or $50 \%$ ) of the patients with microcytic anemia had grade I peptic esophagitis. The table 3 details the distribution of anemic patients according to esophageal pathology and type of anemia(Table 3 ).

Table 3 Distribution of patients according to pathologies of oesophagus and type of anemia

\begin{tabular}{llllllll}
\hline \multirow{2}{*}{ Parameters } & \multicolumn{2}{l}{ Microcytic anemia } & \multicolumn{2}{l}{ Normocytic anemia } & Macrocytic anemia & Total \\
\cline { 2 - 8 } & $\mathbf{n}$ & $\%$ & $\mathbf{n}$ & $\%$ & $\mathbf{n}$ & $\%$ & \\
\hline Peptic I rankOesophagitis & 20 & 50 & 4 & 57,14 & 2 & 50 & 26 \\
Hiatal hernia & 17 & 42,50 & 3 & 42,86 & 2 & 50 & 22 \\
Barrett'soesophagus & 2 & 5 & 0 & 0 & 0 & 0 & 2 \\
Mycoticoesophagitis & $\mathrm{I}$ & 02,50 & 0 & 0 & 0 & 0 & 1 \\
Total & 40 & 100 & 7 & 100 & 4 & 100 & 5 I \\
\hline
\end{tabular}

n, number of case

We found 32 gastric pathologies including 26 gastropathies $(81.25 \%)$. The majority of gastropathies (20 cases/26, 76.92\%) were associated with microcytic anemia. The Table 4 shows the distribution of gastric pathologies according to type of anemia.Most patients (42 Table 4 Distribution of gastric pathologies according to type of anemia cases/44 or $95.45 \%$ ) with microcytic anemia had chronic atrophic gastritis. The majority of gastropathies ( 17 cases/27 or $62.96 \%$ ) were characterized by epigastralgia. Gastropathies were associated with $H$. pylori in 22 cases/26 (84.61\%).

\begin{tabular}{lllllll}
\hline \multirow{2}{*}{ Parameters } & \multicolumn{2}{l}{ Microcytic anemia } & Normocytic anemia & Macrocytic anemia & Total \\
\cline { 2 - 7 } & $\mathbf{n}$ & $\%$ & $\mathbf{n} \%$ & $\mathbf{n}$ & $\%$ & \\
\hline Gastropathy & 20 & 76,92 & 03100 & 3 & 100 & 26 \\
Gastricpolyp & 3 & 11,53 & 0000 & 0 & 0 & 3 \\
Gastriculcer & 2 & 07,70 & 0000 & 0 & 0 & 2 \\
Ulcero-buddingdismutosis & $\mathrm{I}$ & 03,85 & 0000 & 0 & 0 & 1 \\
Total & 26 & 100 & 03100 & 3 & 100 & 32 \\
\hline
\end{tabular}

$\mathrm{n}=$ number of case, $\mathrm{p}=0.0985$ 
We found 05 bulbar pathologies, including 03 ulcers equally distributed between the three types of anemia (microcytic, normocytic, macrocytic) in the under 20,50-60 and over 70 age groups. We also found a bulbar polyp in a 25 -year-old patient with microcytic anemia and bulbar disease in a 63-year-old patient presenting a normocytic anemia.

\section{Discussion}

Any interpretation of our results must take into account the limits of our study, which included selection bias: patients treated in private clinics or by traditional medicine, or in other university hospitals; 36 files were excluded because the examinations confirming the diagnosis were not found. The prevalence is most probably underestimated if we consider the scarcity or even absence of hematologists in rural areas, the poverty of rural populations who often find it difficult to come in town for consultation (Ouagadougou, Bobo-Dioulasso), to pay for complementary examinations and the short duration of our study. Failure to perform a number of specialized paraclinical tests such as the soluble transferrin receptor (sTfR) blood test that can reveal a hidden martial deficiency may lead to an underestimation of the prevalence of our martial deficiencies. Indeed, ferritinemia increases in the event of inflammation, cytolytichepatopathy and hemolysis. In these situations, where ferritinemia may appear falsely normal or even increased, it is necessary to perform the soluble transferrin receptor (sTfR) blood test, which is increased in the event of martial deficiency and not influenced by the presence or absence of an inflammatory syndrome. ${ }^{7,8}$

Over the 17-month period of the study, we collected a total of 58 patients, for a recruitment rate of 40.94 cases/year. This recruitment rate is higher than that of Andrèsand colleagues, ${ }^{7}$ who found 20 patients with anemia over a 5 -year period, for a recruitment rate of 8.33 cases/year. This could be due to the fact that their study was conducted exclusively in elderly subjects. This could be explained by a longer recruitment period than ours and by their contexts. There were more females in all age groups except for those over 60 years of age. Our result is similar to that of Zinebi and colleagues ${ }^{9}$ who found that women outnumbered men in all age groups except those over 70 years of age. At menopausal age (absence of menstruation, pregnancy and breastfeeding), women are less likely to be anemic than women during genital activity. According to the literature, ${ }^{10,11}$ in elderly patients, especially in men, the causes of anemia are mostly digestive lesions.

In our study, asthenia was the most common clinical manifestation of anemia ( 27 cases $/ 58$ or $46.55 \%$ ) just as in the study by Zinebi and colleagues $^{9}$ who found: $41 \%$. Asthenia is one of the earliest and most consistent signs of anemia. ${ }^{12}$ Asthenia, pallor, dizziness, dyspnea and neurosensory signs (tinnitus, paresthesias) were observed in our patients. These results are consistent with the literature ${ }^{7,13,14}$ which indicates the elements of the anemic syndrome (pallor, dizziness, palpitations, asthenia, dyspnea, neurological signs) as a frequent reason for consultation.

The majority of our patientseither 36 cases $/ 58$ or $62.1 \%$ of the patients had microcytic anemia. With the lack of financial resources, ferritinemia had been performed in only $50 \%$ of them. In $50 \%$ of the cases, it was lowered to less than $10 \mu \mathrm{g} / \mathrm{ml}$, suggesting iron deficiency anemia. Nevertheless, martial deficiency could not be ruled out in patients with normal ferritinemia because of our context of aggressions (low level of hygiene, low sanitation) repeated by all kinds of infectious agents (bacteria, viruses, fungi, parasites). This leads to a chronic inflammatory condition which can increase ferritinemia, even in a case of martial deficiency. Moreover, since martial deficiency is the leading cause of anemia in the world and especially in countries with limited resources such as Burkina Faso, ${ }^{12}$ it is very likely that it is present in patients with microcytic anaemia who have not been able to measure ferritinemia. Furthermore, pure inflammatory anemia is typical of subjects over 60 years of age because of the numerous comorbidities. ${ }^{12}$ As a result, its prevalence is lower in our population under study, which is mostly young. Other authors ${ }^{9,14,15}$ also found a majority of patients with microcytic anemia $(56 \%, 60 \%$ and $39 \%$ respectively). Iron deficiency anemia was predominant in all age groups, especially in women under 50 years of age. Women under 50 years of age were the majority of patients in the study with 36 cases/58 (62.1\%). According to the work of Ruivard $\mathrm{M}$ and colleagues, the most affected population groups are nonmenopausal women (menstruation, pregnancy, breastfeeding) and children in countries with limited resources due to unfavorableliving standardsresulting in an unbalanced diet. ${ }^{16}$ However, iron deficiency is frequent even with good nutritional condition, according to the study by Marcoand colleagues, who estimates that $20 \%$ of American women have a low iron reserve and half of them have hypochromic hyposidemicanemia. ${ }^{17}$ Five patients had macrocytic anemia, three of which were vitamin B12 deficient. The etiologies of these deficiencies are dominated by Biermer's disease and by the syndrome of nondissociation of vitamin B12 from its carrier proteins. ${ }^{18}$ This syndrome is the main mechanism of vitamin B12 deficiency in the elderly. ${ }^{19} \mathrm{In}$ our study we found the presence of antibodies against intrinsic factor in 3 of our patients, which suggested Biermer's disease. Folic acid levels were reduced to less than $5 \mu \mathrm{g} / \mathrm{L}$ in two of them with vitamin B9 deficiency. The staple food made of thoroughly cooked cereals (millet, corn) and stews from our populations could explain our results; indeed, our patients could have a deficiency in folate intake because folates are thermolabile vitamins.

Esophagitis (peptic and mycotic) accounted for 52.9\% of esophageal pathologies found in our patients. Mbika Cardorelleand colleague ${ }^{20}$ also found a high proportion of esophagitis in esophageal pathology (61.5\%) although their studies were conducted in nonanemic patients. Zinebiand colleagues ${ }^{9}$ noted a proportion of $33.33 \%$ which was lower than ours; this could be explained by the fact that in their study, upper GI endoscopy was performed only in patients with iron deficiency anemia.

Gastropathies (26 cases/32) accounted for $81.2 \%$ of gastric pathologies. This frequency is close to that of the study by Zinebiand colleagues ${ }^{9}$ which was $75 \%$. The etiologies of gastropathies are multiple, dominated in France ${ }^{21}$ by the consumption of alcohol, tobacco and gastro aggressive drugs. In Burkina Faso, a country with limited resources, poverty pushes many patients to self-medicate with cheaper illegal street drugs. Other etiologies include the consumption of traditional decoctions which sometimes turn out to be gastrotoxic and the consumption of fake alcohol, which is financially and geographically accessible to an idle youth in search of thrills.

There were a total of 5 bulbar pathologies: a bulbar ulcer in the under-20, 50-60 and over-70 age groups, a polyp in the 20-30 age group and a bulbopathy in the 60-70 age group. Bulbar ulcer was predominant in duodenal pathology with $3 / 5$ cases $(60 \%)$. However, its prevalence remains very variable in the literature $20,22,23$ where bulbopathy is sometimes predominant. 


\section{Conclusion}

Diseases of the upper digestive tract diagnosed by endoscopy and histology (especially Pylori $H$ infection) can cause iron-deficiency anemia. In order of frequency, the pathologies of the upper digestive tract most associated with anemia were: gastropathies, esophagitis (peptic and mycotic) and bulbar ulcers. Gastropathies were associated with anemia of deficiency origin, mainly in iron and more rarely in vitamin B12.

\section{Acknowledgments}

None.

\section{Conflicts of interest}

The author declares no conflict of interest.

\section{References}

1. Beutler E, Waalen J. The definition of anemia: what is the lower limit of normal of the blood hemoglobin concentration? Blood. 2006;107(5):17471750 .

2. WHO. Haemoglobin concentrations for the diagnosis of anaemia and assessment of severity: Vitamin and Mineral Nutrition Information System. Geneva, World Health Organization. 2011.

3. McLean E, Cogswell M, Egli I, et al. Worldwide prevalence of anemia, WHO Vitamin and Mineral Nutrition Infromation System, 1993-2005. Public Health Nutr. 2009;12(4):444- 454.

4. Schaefer R M, Huch R, Krafft A. Current recommendations for the treatment of iron deficiency anemia. Rev Med Suisse 2007;3(105):874880 .

5. Nafil H, Tazi I, Sifessalam M, et al. Clinical, biological and therapeutic profile of anemia by vitamin B12 deficiency in the department of hematology of Marrakech (Morocco). Bull Soc Pathol Exot. 2013;106(2):83-88.

6. Lamarque D, Burucoa C, Courillon-Mallet A, et al. Recommendations for the treatment of Pylori $\mathrm{H}$ infection in adults. Hépato-Gastro Oncol Dig. 2017;24:157-170.

7. Andrès E, Mecili M. Letter on the article: How to explore iron-deficiency anemia. Press Med. 2011;40(2):225.

8. Samaha E, Cellier C. Investigation and management of iron deficiency anemia. Hépato-Gastro Oncol Dig. 2014;21:760-772.
9. Zinebi A, Eddou H, Moudden KM, et al. Etiological profile of anemia in a department of internal medecine. Pan Afr Med J. 2017;26:10.

10. Coban E, Timuragaoglu A, Meric M. Iron Deficiency Anemia in the Elderly: Prevalence and Endoscopic Evaluation of the Gastrointestinal Tract in Outpatients. Acta Haematol. 2003;110(1):25-28.

11. Nahon S. Diagnosis strategy of iron deficiency anemia in the elderly. Lett Hépato-Gastroentérologue. 2008;XI:226-29.

12. Andrès E. Iron deficiency anemia: etiology and management, what's new in 2012? HEGEL. 2012; II: 16-26.

13. Belgith A, Mahjoub S, Ben Romdhane N. Etiological study of vitamin B12 deficiency in a Tunisian population. Rev Med. 2009;10:153.

14. Ben Ahmed I, Ben Dahmen F, Ben Amor A, Chabchoub Blouza S. Macrocytic anemia in internal medicine: a study of 30 Tunisian patients. Diabetes Metab. 2011;37:A56.

15. El Hioui M, Ahami T, Aboussaleh Y, et al. Anemia in a Morrocan hospital setting: typology and influence of socio-demographic factors on its incidence. Antropo. 2006;12:83-91.

16. Ruivard M. Iron deficiency anemia in adults: diagnosis and treatment. Nutr Clin Métab. 2017;31:104-106.

17. Aguenaou H. Invisible malnutrition or hidden hunger in Morocco: the strategies to reduce it. Biomatec Echo. 2007;5:158-164.

18. Andrès E, Affenberger S, Vinizio S, et al. Vitamin B12 deficiency in adults: etiology, clinical manifestations and etiologies. Rev Med Int. 2005;26:938-946.

19. Andrès E, Perrin AE, Kraemer JP, et al. Anemia caused by vitamin B 12 deficiency in subjects aged over 75 years: new hypotheses. A study of 20 cases. Rev Med Int. 2000;21(11):946-954.

20. Mbika Cardorelle A, Missambou Mandilou S, Okoko A. Upper GI endoscopy in a pediatric setting in Brazzaville. MAN. 2011;58:277-280.

21. Canard J. A 2 day-endoscopy in France. Results from the 2004 survey. Complete data. La lettre de la SFED. 2005;27:252-268.

22. Djibril M, M'ba K, Kaaga Y, et al. Upper GI endoscopy in an African rural setting in Togo. Med Trop. 2009;69(1):48-50.

23. Rudelli A, Vialette G, Brazier F, et al. Pylori $H$ and gastroduodenal lesions in 547 symptomatic young adults. Gastroentérol Clin Biol. 1996;20(4):379-393. 